Mathematical Research Letters 8, 605-611 (2001)

\title{
A GRADED SYZYGY THEOREM IN MIXED CHARACTERISTIC
}

\author{
E. G. Evans and Phillip Griffith
}

\begin{abstract}
About twenty years ago we gave the original proof of the so-called "syzygy theorem" [6], at least in the case of a local integral domain containing a field. In the intervening years several different styles of proofs and generalizations have appeared (e.g., see our monograph [7, Chapter 3]). Perhaps the most notable of these were the characteristic $p$ proof of Hochster and Huneke [12] and the generalization by Bruns [2] in which the minimal free complex is allowed some positive homology (see [3, 9.5.5]). In addition Dutta [5] and Hochster [11] combined to show that the crucial argument in [6] which rested upon the existence of maximal Cohen-Macaulay modules could be reduced to an application of the weaker canonical element theorem which holds in the equicharacteristic setting (see also Ogoma [13]).

Our original proof [6] relied heavily on the fact we were able to show order ideals of minimal generators for $k$ th syzygies of finite projective dimension must have grade at least $k$. We have long known that a single minimal generator with this property would suffice. Until now we remained unaware of any context in which one could actually apply this (no doubt well-known) fact. However, a recent examination of a graded version of the syzygy theorem in mixed characteristic produced just such a situation. To be specific, we are able to show (see Theorem 8 and Corollary 9), under suitable conditions, a nonfree graded $k$ th syzygy of finite projective dimension over a graded ring $R=R_{0} \oplus R_{1} \oplus R_{2} \oplus \cdots$ must have rank at least $k$, where the ring $R_{0}$ is a discrete valuation ring. Other than the observation described above, our techniques are simply a straightforward blend of those from our original manuscript and standard facts about graded rings and modules. In order to keep the exposition somewhat self contained, we supply a few elementary observations which can be found in some form in our articles [6], [7], [8] or Bruns-Herzog [3, Chapter 9]. These observations or facts about syzygies have been tailored to the context of graded modules over $R=R_{0} \oplus R_{1} \oplus R_{2} \oplus \cdots$ as described above.
\end{abstract}

\footnotetext{
Received April 4, 2001.

Revised version received June 5, 2001.

1991 Mathematics subject classification. Primary: 13B10, 13B15, 13D10, 13F40, Secondary: 13F40, 13H10, 13N05, 14B07

Key Words and Phrases: Cohen-Macaulay local rings, normal domains, ramification, deformations, Segre products.

The authors would like to thank the referee for several corrections and helpful suggestions.
} 
Preliminaries. Let $R$ be a commutative Noetherian ring and let $M$ be a finitely generated $R$-module. For $k \geq 0$, we say that $M$ is a $k$ th syzygy if there is a projective acyclic complex $\cdots \rightarrow F_{s} \rightarrow \cdots \rightarrow F_{1} \stackrel{\varphi_{1}}{\longrightarrow} F_{0} \stackrel{\varphi_{0}}{\longrightarrow} 0$ such that $M \cong \operatorname{coker} \varphi_{k+1}$. In case $R$ is local or graded (and $H_{0}\left(F_{\bullet}\right)$ is graded), then it suffices to consider acyclic free resolutions. The notation $\operatorname{pd}_{R} M<\infty$ indicates that $M$ has finite projective dimension. We say that $M$ satisfies the Serre condition $S_{k}$ if

$$
\operatorname{depth}_{R_{P}} M_{P} \geq \min (k, \operatorname{codim} P)
$$

for each prime ideal $P \in \operatorname{Spec} R$. The $R$-dual of $M$ is denoted by $M^{*}=$ $\operatorname{Hom}_{R}(M, R)$. If $e \in M$, then the order ideal of $e, O_{M}(e)$, is defined by

$$
O_{M}(e)=\left\{f(e) \mid f \in M^{*}\right\} .
$$

We observe that the element $e$ induces an $R$-homomorphism $e: M^{*} \rightarrow R$ defined by $e(f)=f(e)$. Moreover, the image of this homomorphism is $O_{M}(e)$. When $M$ has finite projective dimension and, in addition, $M$ has a finite free resolution (as will always be the case in this article) then the rank of $M$ may be defined as the alternating sum of the ranks in any finite free resolution of $M$.

For the final portion of this discussion we assume that $R=R_{0} \oplus R_{1} \oplus R_{2} \oplus \cdots$ is an $\mathbb{N}$-graded ring ( $\mathbb{N}=$ nonnegative integers) in which $R_{0}$ is a local ring. Actually, our main interest here will concentrate on the case where $R_{0}$ is a DVR (i.e., discrete valuation ring). For such an $R$, there is a unique graded maximal ideal $m=m_{0}+R_{+}$where $m_{0}$ is the maximal ideal of $R_{0}$ and $R_{+}=\oplus_{i>0} R_{i}$. If $M=M_{0} \oplus M_{1} \oplus \cdots$ is a nonzero graded $R$-module, we may assume $M_{0} \neq 0$ (by "twisting" $M$ ) as long as the actual grading is not important. We refer to an element $e \in M-m M$ as a minimal generator of $M$. This is equivalent to the statement that the element $e$ is part of a minimal set of homogenous generators. This is consistent with conventional terminology in the local case (see [7, p. 2]). We remark that, if $m \in M$ is homogenous, then its order ideal $O_{M}(m)$ is graded as well (although the elements of degree zero may all be zero in this case).

Finally, any unexplained terminology may be found in Bruns-Herzog [3] or our monograph [7].

The Graded Syzygy Theorem in Mixed Characteristic. Throughout the remainder of this article the ring $R=R_{0} \oplus R_{1} \oplus R_{2} \oplus \cdots$ is a Noetherian graded ring where $R_{0}=V$ is a $D V R$ with uniformizing parameter $p$. Further, we assume $p$ is a regular element on $R$, that is, the $V$-modules $R_{i}$, for $i>0$, are finitely generated, torsion free modules (we discuss the situation where $p$ is a zero divisor at the end of the article). Although our central point of interest concerns the situation $V$ has mixed characteristic, actually any $D V R$ will do. The graded maximal ideal of $R$ is denoted by $m=p V+R_{+}$. For a graded module $M$, the notation $\bar{M}$ denotes $M / p M$; so also $\bar{R}=R / p R$. Of course $\bar{M}$ is a graded $\bar{R}$-module. 
We begin with a sequence of elementary lemmas concerning grade and codimension of order ideals of homogenous elements. While our statements are for the graded case, it goes without saying that these observations hold in the local case as well. The first of these lemmas is a simple fact about colon ideals. We omit its proof.

Lemma 1. Let $(R, m)$ be as above and let $I$ and $J$ be homogenous ideals such that $I \subseteq J$. If $\operatorname{codim}(I: J) \geq k$, then $\operatorname{codim} I \geq \min (k, \operatorname{codim} J)$.

Lemma 2. Let $M=M_{0} \oplus M_{1} \oplus \cdots$ be a graded kth syzygy of finite projective dimension and let $e \in M_{0}-p M_{0}$. Let $\bar{e}=e+p M \in \bar{M}$. Then one has the following inequalities

$$
\operatorname{codim}_{R} O_{M}(e) \geq \operatorname{codim}_{\bar{R}}\left(O_{M}(e)+(p) /(p)\right) \geq \min \left(k, \operatorname{codim}_{\bar{R}} O_{\bar{M}}(\bar{e})\right)
$$

Proof. The element $e$ induces an $R$-homomorphism $e: M^{*} \rightarrow R$ and similarly $\bar{e}$ induces a homomorphism $\bar{e}: \bar{M}^{*} \rightarrow \bar{R}$. Moreover, one has the following commutative diagram

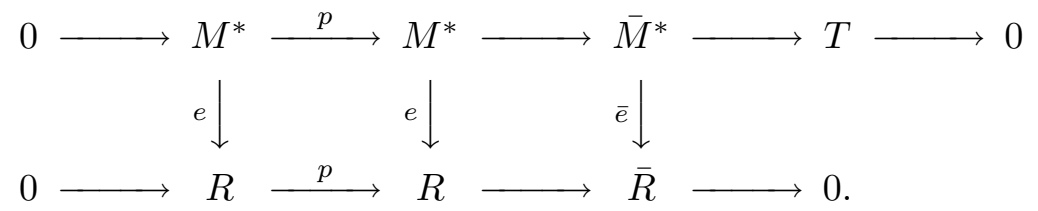

We note that the module $T$ is a result of the fact $\overline{M^{*}} \hookrightarrow \bar{M}^{*}$ is not always an isomorphism; $T$ represents the cokernel. Moreover, $T$ fits into the left exact sequence

$$
0 \rightarrow T \rightarrow \operatorname{Ext}_{R}^{1}(M, R) \stackrel{p}{\rightarrow} \operatorname{Ext}_{R}^{1}(M, R) .
$$

Since $M$ is a $k$ th syzygy of finite projective dimension the $R$-module $\operatorname{Ext}_{R}^{1}(M, R)$ has no support in codimension $\leq k$. Therefore, $\operatorname{codimension}_{R}\left(\operatorname{ann}_{\operatorname{Ext}_{R}}^{1}(M, R)\right) \geq$ $k+1$. Since $T \subseteq \operatorname{Ext}_{R}^{1}(M, R)$ and $p T=0$ it follows $\operatorname{codim}_{\bar{R}}(\operatorname{ann} T) \geq k$. Let $\mathfrak{a}=\operatorname{ann}_{\bar{R}} T$. Then

$$
\mathfrak{a} O_{\bar{M}}(\bar{e}) \subseteq O_{M}(e)+(p) /(p) .
$$

From Lemma 1 we have

$$
\operatorname{codim}_{\bar{R}}\left(O_{M}(e)+(p) /(p)\right) \geq \min \left(k, \operatorname{codim}_{\bar{R}} O_{\bar{M}}(\bar{e})\right) .
$$

Finally an application of Krull's principal ideal theorem yields

$$
\operatorname{codim}_{R} O_{M}(e) \geq \operatorname{codim}_{\bar{R}}\left(O_{M}(e)+(p) /(p)\right) .
$$


Corollary 3 (Notation as above). If $p$ belongs to a prime divisor of $O_{M}(e)$ of minimal codimension, then

$$
\operatorname{codim}_{R} O_{M}(e) \geq 1+\min \left(k, \operatorname{codim}_{\bar{R}} O_{\bar{M}}(\bar{e})\right) .
$$

Proof. This statement follows from Lemma 2 and the observation

$$
\operatorname{codim}_{R} O_{M}(e)=1+\operatorname{codim}_{\bar{R}}\left(O_{M}(e)+(p) /(p)\right) .
$$

Corollary 4. If $M$ satisfies the Serre condition $S_{k}$, then so does $R$. Moreover, the notation "codimension" in (3) may be replaced by "grade", that is,

$$
\operatorname{grade}_{R} O_{M}(e) \geq 1+\min \left(k, \operatorname{grade}_{\bar{R}} O_{\bar{M}}(\bar{e})\right) .
$$

Proof. That $R$ satisfies $S_{k}$ follows directly from the Auslander-Buchsbaum formula pd $M+\operatorname{depth} M=\operatorname{depth} R$. Then since $R$ is $S_{k}$ one has $\operatorname{grade}_{R} I=$ $\operatorname{codim}_{R} I$ for ideals $I$ up to codimension $\leq k$. The ring $\bar{R}$ satisfies these assumptions for ideals of codimension $\leq k-1$.

Our next result gets to the heart of the connection between order ideals and $k$ th syzygies of finite projective dimension. We recall from $[7,3.8]$ that $k$ th syzygies of finite projective dimension coincide with $S_{k}$-modules of finite projective dimension in case the ring $R$ satisfies the Serre condition $S_{k}$.

Proposition 5. Let $M$ be a finitely generated graded $S_{k}$-module of finite projective dimension over $R$. If $e \in M_{0}-p M_{0}$ is such that

$$
\operatorname{grade} O_{M}(e) \geq k>\operatorname{rank} M
$$

then $M / R e$ is an $S_{k-1}$ module of finite projective dimension and $\operatorname{rank}(M / R e)=\operatorname{rank} M-1$.

Proof. If $\mathfrak{p} \in \operatorname{Spec} R$ and $\mathfrak{p} \nsupseteq O_{M}(e)$, then $M_{\mathfrak{p}} \cong R_{\mathfrak{p}} e \oplus N_{\mathfrak{p}}$ where $N=M / R e$. Hence $N_{\mathfrak{p}}$ is an $S_{k}$-module (so also $S_{k-1}$ ) in this case. If $\mathfrak{p} \supseteq O_{M}(e)$, then codim $\mathfrak{p} \geq k$. The exactness of

$$
0 \rightarrow R_{\mathfrak{p}} e \rightarrow M_{\mathfrak{p}} \rightarrow N_{\mathfrak{p}} \rightarrow 0
$$

together with the Depth Lemma [7, p. 13] will give

$$
\operatorname{depth} N_{\mathfrak{p}} \geq \operatorname{depth} M_{\mathfrak{p}}-1 \geq k-1,
$$

once we have established that the $R$-homomorphism $R \rightarrow R e$, where $1 \mapsto e$, is injective. However, if $I$ denotes the kernel of this homomorphism, then $I O_{M}(e)=0$ which means $I=0$ since grade $O_{M}(e)>0$. 
Theorem 6. Let $M=M_{0} \oplus M_{1} \oplus \cdots$ be a finitely generated nonfree $R$-module such that $M$ has finite projective dimension and satisfies the Serre condition $S_{k}$. If $e \in M_{0}-p M_{0}$ is such that $p$ is contained in a prime divisor of $O_{M}(e)$ of minimal codimension, then rank $M \geq k$.

Proof. We assume the contrary, i.e., rank $M<k$. Further, we may assume our " $k$ " is the smallest positive integer for such an occurrence. In this circumstance we note $\bar{M}=M / p M$ is an $S_{k-1}$ module of finite projective dimension over $\bar{R}$. Moreover, since the graded ring $\bar{R}$ must contain a field, we may apply the original syzygy theorem $[7,3.15]$ after making a straightforward translation from the local to the graded case. What we obtain here is that $\operatorname{grade}_{\bar{R}} O_{\bar{M}}(\bar{e}) \geq k-1$ since $\bar{M}$ is necessarily nonfree as an $\bar{R}$-module. Therefore $\operatorname{grade}_{R} O_{M}(e) \geq k$ follows from Corollary 4 above. We invoke Proposition 5 to get that $N=M / R e$ is an $S_{k-1}$-module having rank $<k-1$. This contradicts our original choice of $k$.

Our next move will be to demonstrate that Theorem 6 comes into play in the context we described at the outset. Before doing so we make a few observations about what occurs when one inverts " $p$ ". Let $K$ be the fraction field of $V$. Then $R\left[p^{-1}\right]=K \oplus R_{1}\left[p^{-1}\right] \oplus R_{2}\left[p^{-1}\right] \oplus \cdots$ is an $\mathbb{N}$-graded ring with $R\left[p^{-1}\right]_{0}=K$. Moreover, $M\left[p^{-1}\right]=M_{0}\left[p^{-1}\right] \oplus M_{1}\left[p^{-1}\right] \oplus \cdots$ is a graded $R\left[p^{-1}\right]$-module. The following technical lemma is key to our final argument.

Lemma 7. Suppose the graded $R$-module $M=M_{0} \oplus M_{1} \oplus \cdots$ is such that $M_{0} \neq 0$ and $M$ is torsion free as a $V=R_{0}$-module, that is, assume $p$ is regular on $M$. Further suppose $M\left[p^{-1}\right]$ is a projective $R\left[p^{-1}\right]$-module. Then $M\left[p^{-1}\right]$ is in fact a free $R\left[p^{-1}\right]$-module. Moreover each $e \in M_{0}-p M_{0}$ is a part of some homogenous, free $R\left[p^{-1}\right]$-basis of $M\left[p^{-1}\right]$.

Proof. Since $M\left[p^{-1}\right]$ is a graded $R\left[p^{-1}\right]$-module, we may identify a finite set of homogeneous elements which become a free basis after localizing at the irrelevant maximal ideal $R\left[p^{-1}\right]_{+}$. To form such a homogenous set we may start with a set of minimal generators of $M_{0}$ which form a basis for the $K=R\left[p^{-1}\right]$ vector space $M_{0}\left[p^{-1}\right]$. It is well known (see [9, Lemma 10.4]) that such a homogenous set is actually a free basis for $M\left[p^{-1}\right]$.

Our main result follows.

Theorem 8. Let $R=V \oplus R_{1} \oplus R_{2} \oplus \cdots$ be a graded $V$-algebra for which the uniformizing parameter $p$ is a regular element. If the graded module $M$ is a nonfree $S_{k}$-module of finite projective dimension, then rank $M \geq k$.

Proof. It suffices to consider $k>0$ and $M_{0} \neq 0$. We suppose to the contrary that rank $M<k$. Since $R\left[p^{-1}\right]$ contains a field, it follows from the original syzygy theorem $[7,3.15]$ that $M\left[p^{-1}\right]$ is a locally free module. In particular, $M\left[p^{-1}\right]$ is projective. So Lemma 7 applies and we obtain an element $e \in M_{0}-p M_{0}$ which forms part of a free basis of $M\left[p^{-1}\right]$. Hence, $1 \in O_{M\left[p^{-1}\right]}(e)$ which is 
equivalent to $p^{v} \in O_{M}(e)$ for some $v>0$. We reach a contradiction after invoking Theorem 6.

Corollary 9. We assume the graded ring $R=V \oplus R_{1} \oplus R_{2} \oplus \cdots$ satisfies the Serre condition $S_{k}$ and that $M$ is a kth syzygy of finite projective dimension. Then rank $M \geq k$ in case $M$ is not free.

Proof. We may assume that $k>0$. The result of Auslander-Bridger [1] (see [7, $3.8]$ ) gives that $M$ is an $S_{k}$-module. The result now follows from Theorem 8 .

Corollary 10. Let $R=V\left[X_{1}, \ldots, X_{n}\right]$ be the polynomial ring in $n$ variables over $V$ with the standard grading. If $M$ is a graded nonfree $k$ th syzygy over $R$, then $\operatorname{rank} M \geq k$.

Proof. Since $p d N \leq n$ for each $R$-module $N$, it follows $k<n$ since $M$ is nonfree. Of course $R$ is Cohen-Macaulay; so the claim follows from Corollary 9.

We wish to comment on the case $p$ is a zero divisor on $R$. Two such situations are easily resolved. If $\operatorname{dim} R=\operatorname{dim} R /(p)$ then the "Improved New Intersection Theorem" (see introduction) holds for $R$ and, thus the syzygy theorem holds as well. If $p$ is contained in an embedded prime component then $R$ cannot satisfy the Serre condition $S_{1}$. So the possibilities of $S_{k}$-modules of finite projective dimension are of little interest.

We close with some remarks on Bruns' generalization of the syzygy theorem (see $[2,9.5]$ ). Bruns considers finite free complexes $F_{\bullet}$ which satisfy his condition $\operatorname{codim} F_{\bullet} \geq 0$. To be more specific, if

$$
0 \rightarrow F_{s} \stackrel{\varphi_{s-1}}{\longrightarrow} F_{s-1} \rightarrow \cdots \rightarrow F_{1} \stackrel{\varphi_{1}}{\longrightarrow} F_{0} \rightarrow 0
$$

is such a complex, then Bruns defines

$$
\operatorname{codim} F_{\bullet}=\inf \left\{\operatorname{codim} I_{r_{i}}\left(\varphi_{i}\right)-i \mid 1 \leq i \leq s-1\right\}
$$

where $I_{r_{i}}\left(\varphi_{i}\right)$ denotes the ideal generated by the $r_{i} \times r_{i}$ minors of a matrix representation of the map $\varphi_{i}$. Bruns refers to " $r_{i}$ " as the "expected rank" of $\varphi_{i}$, that is, the rank predicted by the Buchsbaum-Eisenbud Theorem [4] in case $F_{\bullet}$ is acyclic. Within this framework Bruns [2] shows, if $F_{\bullet}$ is a minimal free complex (over a local ring) such that $\operatorname{codim} F_{\bullet} \geq 0$, then $r_{i} \geq \operatorname{codim} F_{\bullet}+i$, for $i=1, \ldots, s-1$. It would be interesting to know if the Bruns result could be extended to the setting of this article or perhaps to the following situation: let $X$ be a nonsingular projective scheme over $V$ having very ample line bundle $\mathcal{O}(1)$. Suppose $\mathcal{F}_{\bullet}$ is a finite acyclic complex of sheaves on $X$ where each $\mathcal{F}_{i}$ is a direct sum of twists " $\mathcal{O}\left(n_{i j}\right)$ " for each $i$. Does the syzygy theorem hold for such complexes? 


\section{References}

1. M. Auslander and M. Bridger, Stable Module Theory, Mem. Amer. Math. Soc., vol. 94, 1969.

2. W. Bruns, The Evans-Griffith Syzygy Theorem and Bass Numbers, Proc. Amer. Math. Soc. 115(1992), no. 4, 939-946.

3. W. Bruns and J. Herzog, Cohen-Macaulay Rings, Cambridge Studies in Advanced Mathematics 39, Cambridge University Press, 1993.

4. D. Buchsbaum and D. Eisenbud, What makes a complex exact?, J. Algebra 25 (1973), 259-268.

5. S. P. Dutta, On the Canonical element Conjecture, Trans. Amer. Math. Soc. 299 (1987), 803-811.

6. E. G. Evans and P. Griffith, The Syzygy Problem, Ann. of Math. 114 (1981), 323-333.

7. Syzygies, in London. Math. Soc. Lecture Notes Series 106, Cambridge University Press, 1985.

8. __ Order Ideals, Commutative Algebra (Berkely, CA, 1987) 213-225, Math. Sci. Res. Inst. Publ. 15, Springer, New York, 1989.

9. R. Fossum, The Divisor Class Group of a Krull Domain, in Ergebnisse der Mathematik und ihrer Grenzgebiete, Band 74, Springer-Verlag, New York-Heidelberg, 1973.

10. R. Hartshorne, Algebraic Geometry, Graduate Texts in Mathematics, No. 52, SpringerVerlag, New York-Heidelberg, 1977.

11. M. Hochster, Canonical Elements in Local Cohomology Modules and the Direct Summand Conjecture, J. Algebra 84 (1983,), 505-553.

12. M. Hochster and C. Huneke, Tight Closure, Invariant Theory, and the Briançon-Skoda Theorem, J. Amer. Math. Soc. 3 (1990), 31-116.

13. T. Ogoma, A Note on the Syzygy Problem, Comm. Algebra. 17 (1989), 2061-2066.

Department of Mathematics, University of Illinois, 1409 West Green Street, UrBANA, ILLINOIS 61801.

E-mail address: graham@math.uiuc.edu

Department of Mathematics, University of Illinois, 1409 West Green Street, UrBANA, ILLINOIS 61801.

E-mail address: griffith@math.uiuc.edu 